\title{
Dynamical Evolution of $\nu$-cooled Disks Following Compact Binary Mergers
}

\author{
William H. Lee ${ }^{1}$ \\ Instituto de Astronomía, UNAM, Apdo. Postal 70-264, Cd. Universitaria, México; \\ wlee@astroscu.unam.mx
}

Summary. Understanding the dynamical evolution of post-merger accretion disks over a long timescale (comparable to their lifetimes) is essential to determine if these can power short GRBs. Here we present preliminary results of such a study, spanning 0.2 seconds, by using a realistic equation of state and taking into consideration the effects of neutrino cooling (the main agent, given the physical conditions in the disks).

\section{Introduction}

The central engines of cosmological gamma ray bursts remain a mystery, but the veil that shrouds them has begun to lift, following the discovery of X-ray, optical and radio afterglows since 1997. It appears now that long GRBs, lasting more than a few seconds - and the only ones for which we have afterglow observations - originate from the collapse of massive stars. This is based partly on the fact that they are associated with regions of inferred high star formation rates in the putative host galaxies [7]. The exact mechanism by which the GRB is created is not entirely clear, but is most likely driven by hypercritical accretion onto a newly formed black hole following the collapse of the star.

Counterparts to short GRBs $\left(t_{d u r} \leq 2 \mathrm{~s}\right)$ have eluded observations so far, and so there is less information to go on, but it seems plausible to assume that they also originate at cosmological distances. One possibility is that they are the product of compact binary mergers, involving the final coalescence process of a double neutron star binary, or one containing a black hole. The merger timescale of these binaries (after the formation of the two compact objects) is a matter of current investigation and not yet settled $[1,8]$. It could be that the lack of afterglows so far is due to the fact that this class of GRBs go off in a low-density environment (outside the host galaxy), making a counterpart harder to find. This scenario also involves hypercritical accretion onto a supramassive neutron star or a stellar mass black hole, following the tidal disruption of the secondary and the formation of an accretion disk.

In previous work, we have investigated precisely the outcome of the binary merger of a neutron star with a stellar mass black hole, using a 3D hydrodynamics code [3]. The calculation was only carried out for $20 \mathrm{~ms}$ or 

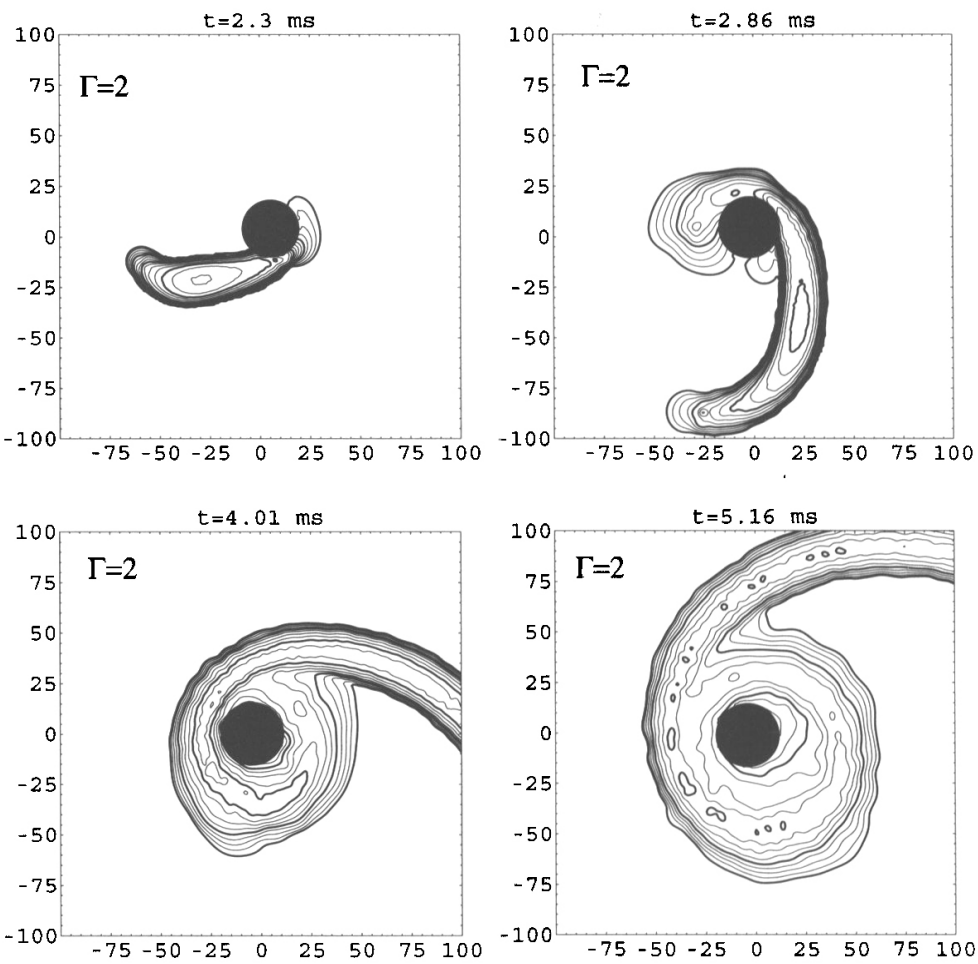

Fig. 1. First stages in the coalescence of a black hole-neutron star binary. The logarithmic contours are for density in the orbital plane. The rapid formation of an accretion structure close to azimuthal symmetry is clear. The axes are labeled in $\mathrm{km}$.

so, due to computational limitations. A typical sequence of events is shown in Fig. 1, for an initial mass ratio $M_{N S} / M_{B H}=0.31$ using an ideal gas equation of state with adiabatic index $\Gamma=2$. After a few orbital timescales, the accretion torus is nearly axisymmetric, contains a few tenths of a solar mass and is typically $400 \mathrm{~km}$ across. We refer the reader to [3] for further details of this calculation.

The dynamical timescale (ms) in such a system is much too short to account for even short GRBs, and its evolution needs to be understood for a longer period. We have addressed this, sacrificing one dimension in the problem by assuming azimuthal symmetry and modeling the disk in cylindrical coordinates $(r, z)$ for $0.2 \mathrm{~s}$. The first results of these calculations, reporied in [4], took the initial conditions from an azimuthal average of the 3D results mentioned above, assumed an equally simple equation of state (ideal gas, $\gamma=4 / 3$ ) and used an $\alpha$-viscosity to account for the transport angular momentum. 

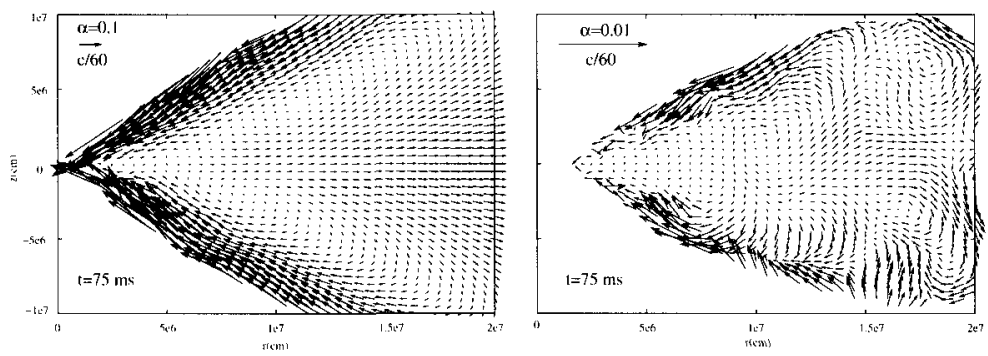

Fig. 2. Velocity field in the inner region of the accretion disk for $\alpha=0.1$ (left) and $\alpha=0.01$ (right) at $t=75 \mathrm{~ms}$. The circulation patterns are clearly seen in both cases.

Here we describe a new set of more realistic calculations for the same initial configurations, having improved both the equation of state, and taking into account the cooling of the disk through neutrino emission.

\section{Post-merger Evolution of the Disk}

\subsection{Physical Conditions and Equation of State}

The accretion structures that are formed following the disruption of the neutron star are typically very dense $\left(\rho \geq 10^{11} \mathrm{~g} \mathrm{~cm}^{-3}\right)$ and hot $(T \geq 5 \mathrm{MeV})$. Nuclei are entirely photodisintegrated, and the gas consists of non-degenerate free neutrons and protons, together with an extremely relativistic degenerate electron gas and photons. With these assumptions the pressure is given by

$$
P=\rho k T / m_{p}+K\left(\rho / \mu_{e}\right)^{4 / 3}+a T^{4} / 3
$$

Note that even though the temperature is well above the threshold for pair creation, the number of these pairs is extremely small because of the degeneracy. Thus they do not contribute to the last term in the above equation. Steady state solutions to this type of disk have been recently considered $[2,5]$. The time dependent evolution however, has not, and is of great importance, since the disk is not being fed matter or angular momentum by any external agent.

The timescale for the gas to achieve $\beta$-equilibrium, $\tau_{\beta}$, is shorter than the accretion timescale, $\tau_{a c e}$. The electron fraction $Y_{e}$ (and immediately also $\mu_{e}$ ) is hence computed by assuming $\beta$-equilibrium, to maintain consistency. The photons cannot cool the gas, since $\tau_{a c c}$ is itself shorter than their diffusion time, $\tau_{\text {dif }}$. However, neutrinos are emitted in copious amounts (our calculations consider neutrino bremsstrahlung, plasmon decay, pair annihilation and electron and positron capture onto nucleons), and are the main source of cooling (the rates are as given in [2]). We do not consider explicitly the effects 


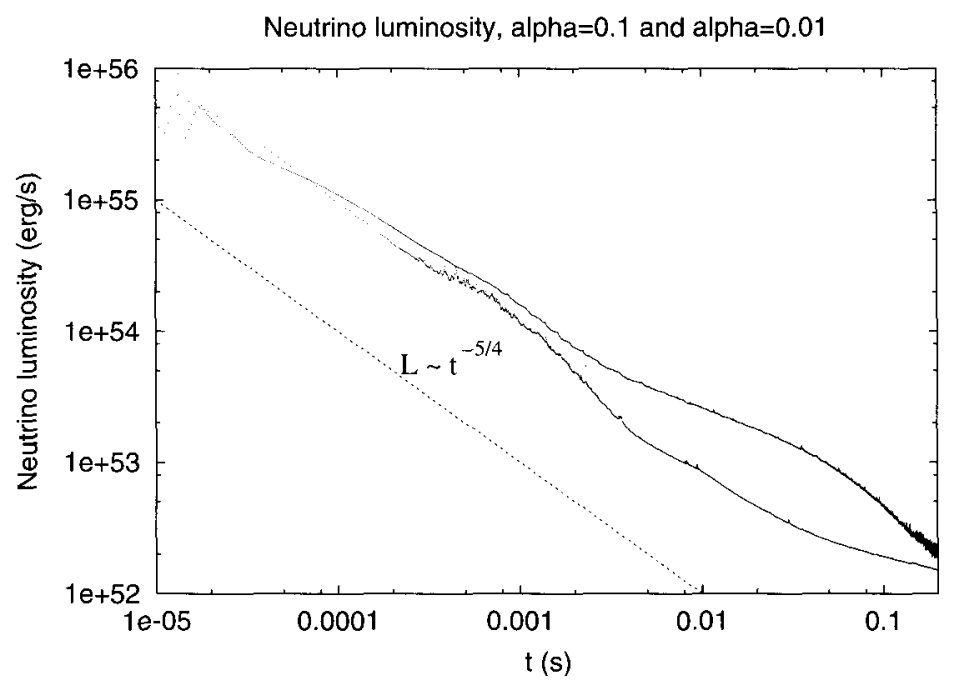

Fig. 3. Neutrino luminosity $L_{\nu}$ as a function of time for $\alpha=0.1$ (upper solid line) and $\alpha=0.01$ (lower solid line). The dashed line is a reference power law $L_{\nu} \propto t^{-5 / 4}$.

of neutrino transport, but simply assume that the optical depth of the fluid is small enough that they can escape freely. A crude a posteriori estimate of where the neutrino-surface lies indicates that $\tau_{\nu} \simeq 1$ at $\rho \simeq 10^{11} \mathrm{~g} \mathrm{~cm}^{-3}$ (based on the scattering off free nucleons (e.g., [6]). So the innermost region of the accretion disk becomes optically thick to neutrinos, and diffusive effects are expected to be important. This is something that clearly needs to be addressed in future work. For now, our assumptions allow us to compute upper limits to the neutrino luminosity in such disks.

\subsection{Numerical Modeling}

The actual dynamical evolution calculations are carried out using the same Smooth Particle Hydrodynamics (SPH) code used in previous studies of such disks [4]. The code solves the equations of motion for the fluid in the presence of a central mass (the black hole) in azimuthal symmetry. The equation of state has been modified, and neutrino cooling has been implemented, according to the discussion in section 2.1. The central mass produces a Newtonian point-mass potential, and the event horizon is modeled by an absorbing boundary at the Schwarzschild radius $r_{g}=2 G M_{\mathrm{BH}} / c^{2}$. The disk accretes because of the transport of angular momentum, modeled with an $\alpha$-viscosity. The disks have initially between 20,000 and $80,000 \mathrm{SPH}$ particles. We show here results for calculations that used $\alpha=0.01$ and $\alpha=0.1$, lasting $0.2 \mathrm{~s}$. 


\subsection{Results}

Figure 2 shows the velocity field in the inner regions of the accretion disks for two values of the viscosity parameter. The circulation patterns that are seen are similar to what we obtained in previous calculations. For large $\alpha$ the circulation has a vertical scale that is comparable to the radial coordinate, while for small $\alpha$ the cells are much smaller. The pattern persists throughout the calculations, diminishing in strength progressively.

Figure 3 shows the neutrino luminosities as functions of time for the same calculations. Roughly, $L_{\nu} \propto t^{-5 / 4}$, with slight differences between the two cases at late times. These are basically due to the fact that the high-viscosity disk dissipates more energy and is thus able to maintain higher luminosities. It is worth mentioning that for the physical conditions in the disk, electron and positron capture onto free nucleons gives the dominant contribution to the cooling rate.

\section{Conclusions}

From the curves shown in Fig. 3 one can in principle compute the energy deposition rates through $\nu-\bar{\nu}$ annihilation, as a function of time, and investigate whether this process can drive a relativistic outflow.

Regardless of this particular aspect, after $0.2 \mathrm{~s}$, the disk still contains several tenths of a solar mass, and densities in the inner regions reach $10^{11} \mathrm{~g} \mathrm{~cm}^{-3}$. Were the magnetic field in such a plasma to reach equipartition values, the field strength would be $B \simeq 10^{15-16} \mathrm{G}$, sufficient to be able to drain the energy of the disk (or of the black hole) and power a short GRB.

Acknowledgement. It is a pleasure to thank W. Kluzniak and E. Ramírez-Ruiz for discussions and collaboration. This work is supported in part by Contract (36632E).

\section{References}

1. N. Ivanova, K. Belczynski, V. Kalogera, F.A. Rasio, R.E. Taam: Astrophys. J. $\mathbf{5 9 2}, 475(2003)$

2. K. Kohri, S. Mineshige: Astrophys. J. 577, 311 (2002)

3. W.H. Lee: Mon. Not. R. Astron. Soc. 328, 583 (2001)

4. W.H. Lee, E. Ramirez-Ruiz: Astrophys. J. 577, 893 (2002)

5. R. Narayan, T. Piran, P. Kumar: Astrophys. J. 557, 949 (2001)

6. S.L. Shapiro, S.A. Teukolsky: In: Black holes, white dwarfs, and neutron stars. (Wiley Interscience: New York, 1983) p. 663

7. J. van Paradijs, C. Kouveliotou, R.A.M.J. Wijers: Ann. Rev. Astron. Astrophys. 38, 379 (2000)

8. R.Voss, T.M. Tauris: Mon. Not. R. Astron. Soc. 342, 1169 (2003) 